\title{
PRESSURE RELIEF
}

\section{Reduced mobility}

can cause

pressure sores.

Nicky Stacey

advises how to

avoid them.
P ressure sores should not be seen as the inevitable result of becoming elderly or disabled. They are painful and can cause someone to spend several months in hospital.

However, many sores could and should be prevented.

It is important to remember that pressure care should be provided right across the range of daily living activities. It is not sufficient only to provide a pressure cushion for the wheelchair, for example. Protection may also be needed in bed, in the bath and on the commode or toilet.

People with normal muscle tone instinctively move when pressure builds up because they experience discomfort. This is often seen as fidgeting. People with limited physical abilities, owing to decreased sensation or mobility may be unable to do this consciously and, as a result, pressure points build up.

\section{CUSHIONS}

There are several points to consider when you come to select a cushion.

- Some cushions are made of substances, e.g. air, water, gel. that move and conform to the shape of the body. This type of cushion distributes pressure well, as it forms a large surface over which areas of high pressure can be dispersed.

These do not provide help when transferring; if the user needs to push down on the cushion to gain some leverage, the contents of the cushion will move as soon as the body weight is lifted and the support will disappear.

If a cushion is too soft or too thin, it will become so compressed that it no longer has any pressure-relieving properties. This is called 'bottoming out'. It causes most of the pressure to focus on small areas, while other areas are offering little or no support. - Some cushions are designed to reduce shear forces as much as possible. Cushions which have individual small balloons making up an egg box-shaped surface or foam cushions which have a surface of foam cubes are able to move with the body so that the 'pull' on the outer layer of skin is decreased.

Cushions which are ramped backwards decrease the likelihood of users sliding forward in the chair.

The user who has poor control of the muscles of the upper body and, as a result, finds it difficult to maintain sitting balance may find that cushions that easily conform to the body and its movements do not feel particularly safe. A more stable cushion may be preferable.

- The adjustment of some cushions requires a certain amount of skill initially, so that they provide the correct amount of pressure relief. - Some cushions have power packs that could go wrong; others may need mending after a puncture or a split. If there is no one able to do this, it may be better to choose a cushion that needs no setting up or maintaining.

The portability of the cushion will be important for people who frequently need to lift it on and off the wheelchair or in and out of the car. Some cushion materials, like gel or water, are heavy and, even with handles on the covers, are not easy to lift. 